\title{
A Note on Starshaped Sets in 2-Dimensional Manifolds without Conjugate Points
}

\author{
Adem Kılıcman and Wedad Saleh \\ Department of Mathematics and Institute for Mathematical Research, Universiti Putra Malaysia (UPM), \\ 43400 Serdang, Selangor, Malaysia \\ Correspondence should be addressed to Adem Kilıcman; akilic@upm.edu.my
}

Received 25 June 2014; Accepted 19 August 2014; Published 28 August 2014

Academic Editor: M. Mursaleen

Copyright ( 2014 A. Kilicman and W. Saleh. This is an open access article distributed under the Creative Commons Attribution License, which permits unrestricted use, distribution, and reproduction in any medium, provided the original work is properly cited.

Let $W^{n}$ be $C^{\infty}$ complete, simply connected $n$-dimensional Riemannian manifolds without conjugate points. Assume that $S \subset W^{2}$ is starshaped where ker $\$ \neq S$. For every point $x \in S \backslash \operatorname{ker} S$, define $A(x)=\{y: y$ lies on some geodesic segment in $S$ from $x$ to a point of $\operatorname{ker} S\}$. There is a finite collection $\mathscr{A}$ of all maximal $A$ sets whose union is $S$. Further, $\operatorname{ker} S=\cap\{A: A$ in $\mathscr{A}\}$.

\section{Introduction}

We begin with some definitions from [1-3]. A subset $B$ in a Riemannian manifold $M$ is convex if, for each pair point $p, q \in B$, there is a unique minimal geodesic segment from $p$ to $q$ and this segment is in $B$. When dealing with a subset $B C$ $W^{n}$, the word "a unique minimal geodesic segment" should be replaced by "the geodesic." A subset $S$ in a Riemannian manifold $M$ is starshaped if there is a point $p \in S$ such that, for all $q \in S$, there is a unique minimal geodesic segment from $p$ to $q$ and this segment is in $S$. The subset of $S$ consisting of all points like $p$ is called the kernel of $S(\operatorname{ker} S)$. In $W^{n}$, a subset $S$ is starshaped if there is a point $p \in S$ such that, for all $q \in S$, the geodesic segment joining $p$ and $q$ is contained in $S$. The subset $S$ is convex if and only if $\operatorname{ker} S=S$. Throughout the paper, $\operatorname{Int}(B)$ and $\partial B$ will denote the interior and the boundary of the subset $B$, respectively. Let $d(p, q)$ denote the distance between the two points $p, q \in W^{n}$. For the pair of points $p, q \in W^{n}, \gamma_{p q}$ will denote the geodesic segment joining $p, q$. For $x \in S$ and $B \subset S, d(x, B)=\inf \{d(x, b): b \in$ $B\}$. The distance to $B \subset S$ is locally maximal at $x \in S$ if there is some neighborhood $U$ of $x$ such that $d(y, B) \leq d(x, B) \forall y \in$ $U \cap S$. Finally, all manifolds, maps, fields, and so forth are discussions to make sense. All curves are parametrized by arc-length.

Readers may refer to [4] for discussions concerning visibility via geodesic segment and starshaped sets. The behavior geodesic in $W^{n}$ has been discussed by many geometrers as Eberlein [2] and Goto [5]. For more properties of convexity and starshapedness in $W^{n}$, see [6-9].

\section{Main Results}

Let $W^{2}$ be 2-dimensional manifolds without conjugate points, and assume that $S \subset W^{2}$ is starshaped. For every $x \in S \backslash \operatorname{ker} S$, we define $A(x)=\left\{y: y \in \gamma_{x k} \subset S, k \in \operatorname{ker} S\right\}$. Clearly $S=$ $\cup\{A(x): x \in S \backslash \operatorname{ker} S\}$.

Lemma 1. Let $S$ be starshaped in 2-dimensional manifolds without conjugate point. Then

(a) the set $\operatorname{ker} S$ is convex,

(b) if $\gamma$ is any geodesic segment in $S$ joining points of $\operatorname{ker} S$, then $\gamma \subset \operatorname{ker} S$,

(c) for every point $x \in S$, there is a unique point $k$ of $\operatorname{ker} S$ closest to $x$; that is, there is a unique point $k$ of $\operatorname{ker} S$ such that $d(x, k)=d(x, \operatorname{ker} S)$.

Proof. We have the following.

(a) Since $S$ is a starshaped subset of $W^{2}$, by [10], $\operatorname{ker} S$ is convex.

(b) Since ker $S$ is convex, clearly any geodesic segment in $S$ joining points of $\operatorname{ker} S$ is in $\operatorname{ker} S$. 
(c) Let $x$ be an arbitrary point in $S$. If $x \in \operatorname{ker} S$, then the result is trivial. $x \in S \backslash \operatorname{ker} S$. Let there be two points $k_{1}$ and $k_{2}$ in $\operatorname{ker} S$ closest to $x$, that is, $d\left(x, k_{1}\right)=d\left(x, k_{2}\right)=d(x, \operatorname{ker} S)$, to show that $k_{1}=k_{2}$. Suppose on the contrary that $k_{1} \neq k_{2}$. Since $k_{1}$ and $k_{2}$ are in $\operatorname{ker} S$, then $\gamma_{k_{1} k_{2}} \subset \operatorname{ker} S$. Let $k \in \gamma_{k_{1} k_{2}} \subset \operatorname{ker} S$, such that $k \neq k_{1}$ and $k \neq k_{2}$. However, $k$ is closer to $x$ than $k_{1}$ and $k_{2}$, contradicting the assumption that $k_{1}$ and $k_{2}$ are closest to $x$; then $k_{1}=k_{2}$.

Proposition 2. Let $S$ be starshaped in 2-dimensional manifolds without conjugate points. For every point $x \in S \backslash \operatorname{ker} S$, one defines $A(x)=\left\{y: y \in \gamma_{x k} \subset S, k \in \operatorname{ker} S\right\}$. For any pair of points $x, z \in S \backslash \operatorname{ker} S$, if $x \in A(z)$, then $A(x) \subseteq A(z)$.

Proof. Let $s \in A(x)$; then $s \in \gamma_{x k}$ where $k \in \operatorname{ker} S$. Since $x \in A(z)$, then $x \in \gamma_{z k}$ where $k \in \operatorname{ker} S$. This implies that $\gamma_{x k} \subset \gamma_{z k}$; then $s \in \gamma_{z k}$. We conclude that $s \in A(z)$ and the proof is complete.

Proposition 3. Let $S \subset W^{2}$ be a starshaped set. Let $x \in S \backslash$ $\operatorname{ker} S$. If $x \notin \partial S$, then there is some point $z \in \partial S$ such that $x \in A(z)$ and hence $A(x) \subset A(z)$. Moreover, $d(x, \operatorname{ker} S)$ is not locally maximal at $x$.

Proof. Let $z \in \partial S$ and $x \notin \partial S$. Let $\gamma_{x k} \subset S$ be a geodesic segment from $x$ to $k$ where $k \in \operatorname{ker} S$. Then $\gamma_{z x} \cup \gamma_{x k}$ is a geodesic segment in $S$ from $z$ to $x$ to $k$. Hence $x \in A(z)$. Then, by Proposition 2, we have $A(x) \subseteq A(z)$. Moreover, $z \in A(z) \backslash A(x)$, because $z \notin A(x)$. Then $A(z) \nsubseteq A(x)$, and $A(x) \subset A(z)$. Finally, it is clear that, for all points $s \in \gamma_{z x}$, $d(s, \operatorname{ker} S)>d(x, \operatorname{ker} S)$. Then the distance to $\operatorname{ker} S$ is not locally maximal at $x$.

Proposition 4. Let $x \in S \backslash \operatorname{ker} S$. If $A(x)$ is a maximal $A$ set, then $d(x, \operatorname{ker} S)$ is locally maximal at $x$.

Proof. Assume on the contrary that every neighborhood of $x$ contains some point $z$ for which $d(x, \operatorname{ker} S)<d(z, \operatorname{ker} S)$. By Proposition 3, let $z \in \partial S$. Then $x \in \gamma_{z x} \cup \gamma_{x k}$ where $k \in$ ker $S$. Then $x \in \gamma_{z x}$, so $x \in A(z)$. By Proposition $2, A(x) \subset$ $A(z)$. Since $A(x)$ is maximal, then $A(z) \subset A(x)$ which means that $z \in \gamma_{x k}$ where $k \in \operatorname{ker} S$, which contradicts $d(x, \operatorname{ker} S)<$ $d(z, \operatorname{ker} S)$

Proposition 5. Let $S \subset W^{2}$ be a starshaped set. Let $x \in S$ such that $d(x, \operatorname{ker} S)$ is locally maximal at $x$. Then

(a) the point $x$ is a vertex of $S$,

(b) for every point $z$ near $x, z \neq x, d(z, \operatorname{ker} S)$ is not locally maximal at $z$,

(c) $A(x) \nsubseteq A(w)$ for any point $w \neq x$.

Proof. We have the following.

(a) Since $d(x, \operatorname{ker} S)$ is locally maximal at $x$, then $x \in$ $S \backslash$ ker $S$ and every neighborhood of $x$ contains some point $z$ such that $d(x, \operatorname{ker} S)>d(z, \operatorname{ker} S)$. By Proposition 3, $x \in \partial S$. Then $x$ must be a vertex of $S$. (b) It is direct from (a).

(c) $x \in A(x) \backslash A(w)$, because $x \notin A(w)$ for any $w \neq x$. Then $A(x) \nsubseteq A(w)$.

Theorem 6. Let $S \subset W^{2}$ be a starshaped set. For every point $x \in S$, there is a finite subcollection $\mathscr{A}$ of all maximal $A$. Then, the following conditions hold:

(a) $S=\cup\{A: A$ in $\mathscr{A}\}$,

(b) for every point $z \in S \backslash \operatorname{ker} S, A(z)$ in $\mathscr{A}$ if and only if $A(z)$ is a maximal $A$ set.

Proof. Assume that $\mathscr{A}=\left\{A_{i}(x): x \in S \backslash \operatorname{ker} S, A_{i}(x) \nsubseteq\right.$ $\left.A_{j}(x) \forall 1 \leq i, j \leq n\right\}$.

(a) For $z \in S, z$ belongs to at least one set $A_{i}$. Either $A_{i}$ is in $\mathscr{A}$ or $A_{i} \subset A_{j}$ where $A_{j}$ is in $\mathscr{A}$. This implies that $z \in \cup\{A: A$ in $\mathscr{A}\}$; then $S \subseteq \cup\{A: A$ in $\mathscr{A}\}$.The reverse inclusion is immediate, so the sets are equal.

(b) $z \in S \backslash \operatorname{ker} S$. Let $A(z)$ be in $\mathscr{A}$; then $A(z)$ is a maximal set. To prove that $A(z)$ is in $\mathscr{A}$, assume on the contrary that $A(z)$ is not in $\mathscr{A}$. Since $z \in S \backslash \operatorname{ker} S$, by (a), there is $A_{i}(w)$ in $\mathscr{A}$ such that $A(z) \subset A_{i}(w)$, but $A(z)$ is a maximal $A$ set; this implies that $A(z)=A_{i}(w)$. Then, $A(z)$ is not subset of any set $A_{i}$, so $A(z)$ is in $\mathscr{A}$.

Lemma 7. Let $S \subset W^{2}$ be a starshaped set. Each point $x \in$ $S \backslash \operatorname{ker} S$. Then

(a) the set $A(x)=\left\{y: y \in \gamma_{x y} \subset S, k \in \operatorname{ker} S\right\}$ is convex,

(b) if $\gamma$ is any geodesic segment in $S$ joining points of $A(x)$, then $\gamma \subset A(x)$.

Proof. We have the following.

(a) Let $k_{1}, k_{2} \in \operatorname{ker} S$; then $\gamma_{k_{1} k_{2}} \subset \operatorname{ker} S$, because $\operatorname{ker} S$ is convex. Moreover $\gamma_{x k_{1}} \cup \gamma_{x k_{2}} \cup \gamma_{k_{1} k_{2}} \subset S$. Let $z_{1}, z_{2} \in$ $A(x)$, such that $z_{i} \in \gamma_{x k_{i}}, i=1,2$, respectively. We will prove that $\gamma_{z_{1} z_{2}} \subset A(x)$. Assume on the contrary that $\gamma_{z_{1} z_{2}}$ is not in $A(x)$; then there is $z \in \gamma_{z_{1} z_{2}}$ and $z \in S \backslash A(x)$. Let $k$ be an arbitrary point of ker $S$ such that $z \in \gamma_{x k}$, but $z \notin A(x)$ which means that $\gamma_{x k}$ is not in $A(x)$, which is a contradiction.

(b) It is clear that any geodesic segment in $S$ joining points of $A(x)$ is in $A(x)$, because $A(x)$ is convex.

Theorem 8. Let $S \subset W^{2}$ be a starshaped set. Then $\operatorname{ker} S=$ $\cap\{A: A$ in $\mathscr{A}\}=\cap\{A(x): x \in S \backslash \operatorname{ker} S\}$.

Proof. Clearly ker $S \subseteq \cap\{A(x): x \in S \backslash \operatorname{ker} S\} \subseteq \cap\{A$ : $A$ in $\mathscr{A}\}$. Now, we will prove that $\cap\{A: A$ in $\mathscr{A}\} \subseteq \operatorname{ker} S$. Let $z_{1} \notin \operatorname{ker} S$; then there is a point $z_{2} \in S$ such that $z_{1}$ does not see $z_{2}$ via $S$. Since $z_{2} \in S$, by Theorem 6 , there is $A_{i}(w)$ in $\mathscr{A}$ such that $z_{2} \in A_{i}(w)$; by Lemma 7, $A_{i}(w)$ is convex; then $z_{1} \notin A_{i}(w)$; this implies that $z_{1} \notin \cap\{A: A$ in $\mathscr{A}\}$; then $\cap\{A: A$ in $\mathscr{A}\} \subseteq \operatorname{ker} S$ and the proof is complete. 


\section{Conclusion}

All results of the present work are valid in Euclidean space $E^{n}$ as manifolds without conjugate points [5], but the generalization of these results to $W^{n}, n \geq 3$, is more difficult and is left as open problem.

\section{Conflict of Interests}

The authors declare that there is no conflict of interests regarding the publication of this paper.

\section{Acknowledgment}

The authors are very grateful to the anonymous referees for their valuable suggestions and comments, which helped the authors to improve this paper.

\section{References}

[1] C. Udriste, Convex Functions and Optimization Methods on Riemannian Manifolds, Kluwer Academic Publishers, 1994.

[2] P. Eberlein, "Geodesic flow in certain manifolds without conjugate points," Transactions of the American Mathematical Society, vol. 167, pp. 151-170, 1972.

[3] W. Erb, Uncertaintry principles on Riemannian Manifolds, Logos, Berlin, Germany, 2010.

[4] J. Cel, "Tietze-type theorem for partially convex planar sets," Bulletin de la Société Royale des Sciences de Liège, vol. 69, no. 1, pp. 17-20, 2000.

[5] M. S. Goto, "Manifolds without focal points," Journal of Differential Geometry, vol. 13, no. 3, pp. 341-359, 1978.

[6] R. J. Dwilewicz, "A short history of convexity, Differential Geometry-Dynamical Systems, vol. 11, pp. 112-129, 2009.

[7] L. Conlon, Differentiable Manifolds, Birkhäuser, Boston, Mass, USA, 2nd edition, 2008.

[8] J. J. O'Sullivan, "Manifolds without conjugate points," Mathematische Annalen, vol. 210, pp. 295-311, 1974.

[9] S. Mendonça and H. Mirandola, "Hypersurfaces whose tangent geodesics do not cover the ambient space," Proceedings of the American Mathematical Society, vol. 136, no. 3, pp. 1065-1070, 2008.

[10] M. Beltagy, “On starshaped sets," Malaysian Mathematical Society. Bulletin. Second Series, vol. 11, no. 2, pp. 49-57, 1988. 


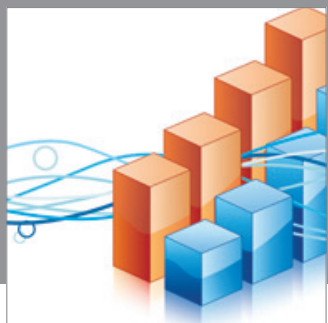

Advances in

Operations Research

mansans

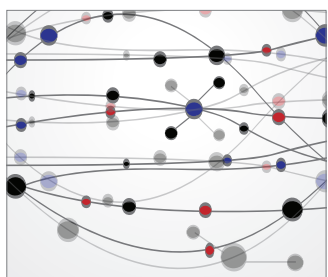

The Scientific World Journal
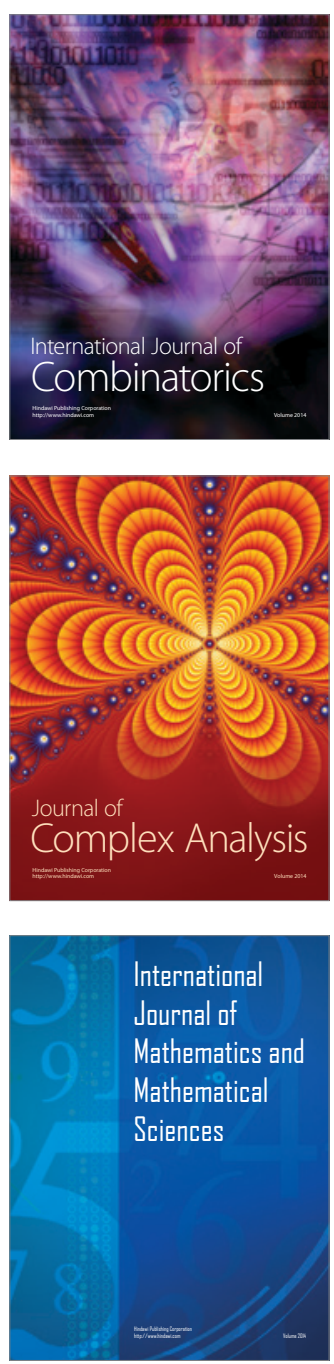
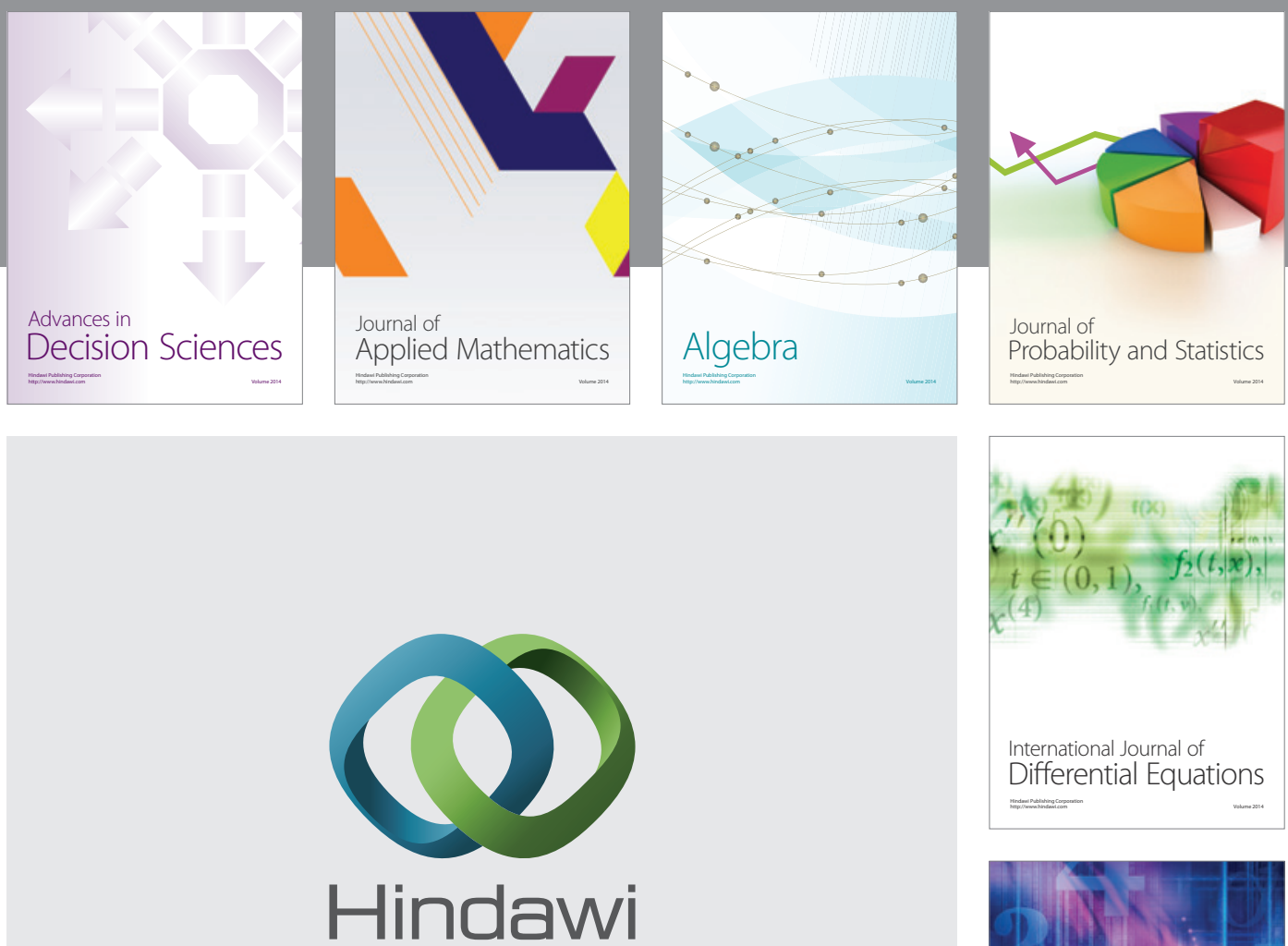

Submit your manuscripts at http://www.hindawi.com
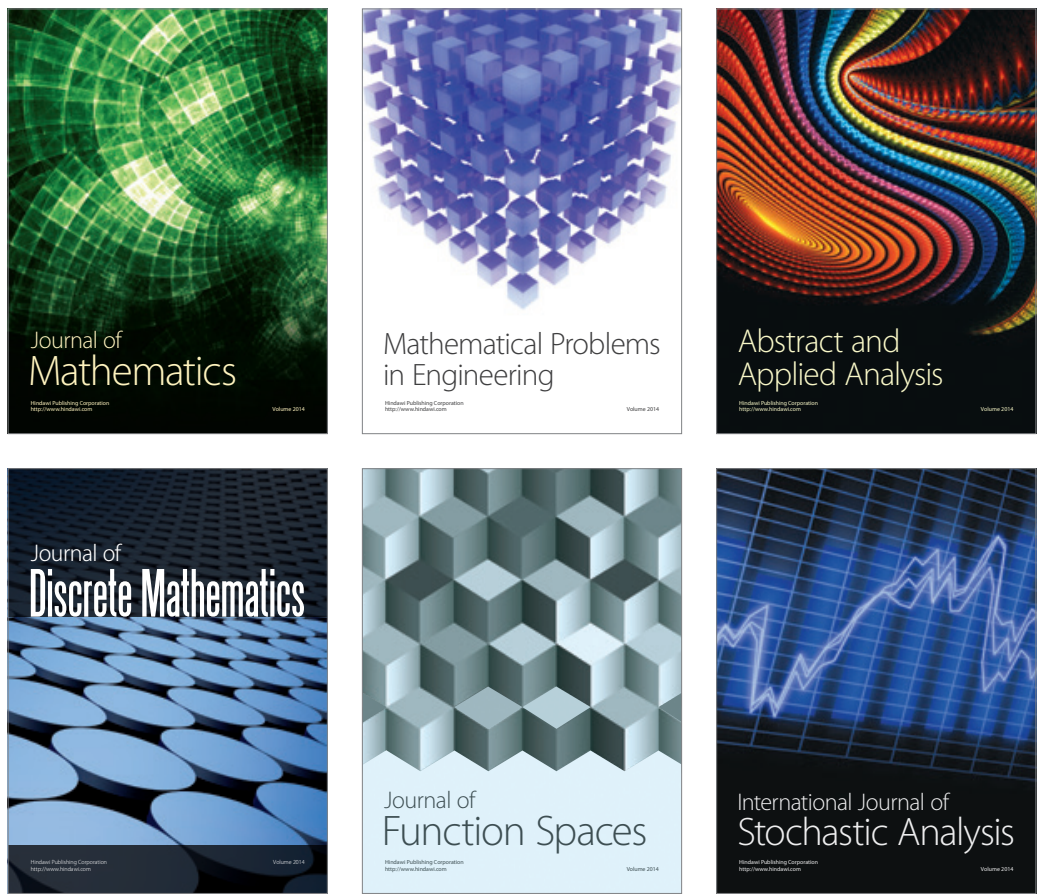

Journal of

Function Spaces

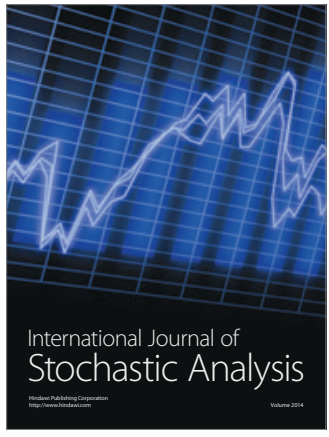

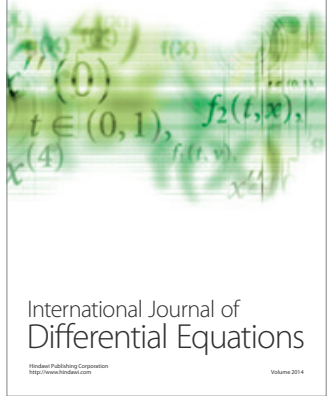
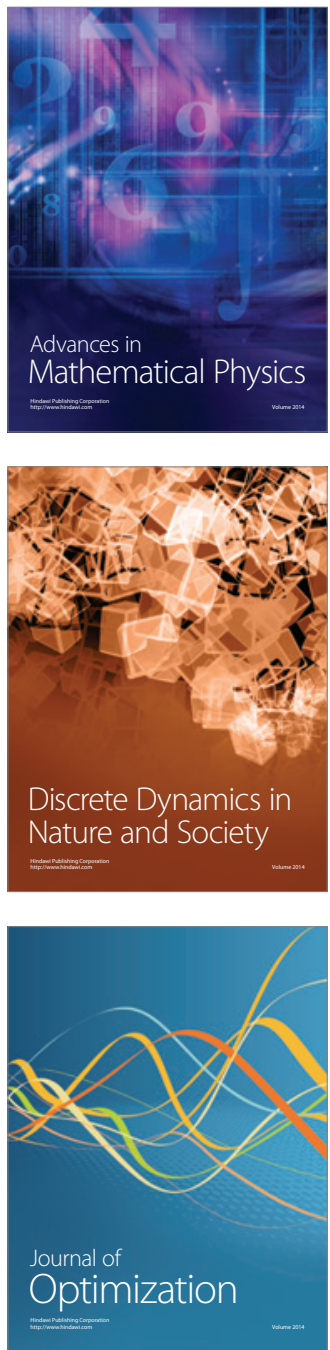